\title{
ON THE UNKNOTTEDNESS OF THE FIXED POINT SET OF DIFFERENTIABLE CIRCLE GROUP ACTIONS ON SPHERES-P. A. SMITH CON JECTURE
}

\author{
BY WU-YI HSIANG
}

Communicated by W. S. Massey, May 18, 1964

The original P. A. Smith conjecture is that there are no $Z_{p}$ actions on $S^{3}$ with a knotted $S^{1}$ as fixed point set. The so-called generalized P. A. Smith conjecture is that there are no $Z_{p}$ or circle group actions on $S^{n}$ with a knotted $S^{n-2}$ as fixed point set [2], [8]. Mazur [5], [6] tried to give counterexamples for the cases $n=4,5$ but there are several mistakes. In this paper, we show that the P. A. Smith conjecture is true for differentiable circle group actions. According to Giffen [3], there are examples of differentiable $Z_{p}$ actions on $S^{n}$, $n \geqq 5, p$ arbitrary, with knotted $S^{n-2}$ as their fixed point sets.

In view of the fact that the cohomological theories for $Z_{p}$ actions and circle group actions are always parallel, it becomes more interesting to find the differences between $Z_{p}$ actions and circle group actions. We will show that the circle group actions are more regular, in a sense, than $Z_{p}$ actions.

Theorem I. Suppose given a differentiable action of $S^{1}$ on $S^{n}, n \neq 4$, with its fixed point set $F=S^{n-2}$, then $F$ is necessarily unknotted. If $n=4$, then $S^{n}-F$ has the homotopy type of a circle. Actually, except for the cases $n=4,5$, the following stronger result is true.

TheOREM I'. A differentiable action of $S^{1}$ on $S^{n}$ with an $(n-2)$ dimensional fixed point set $F$ is orthogonal if and only if $F$ is an (n-2)sphere.

The above theorems are just special cases of the following classification theorem. First, we give a construction.

Construction. Given a compact contractible manifold $X$ of dimension $n-1, n \geqq 5$, we may have a circle group action on the smoothed $D^{2} \times X$ simply by letting $g \cdot(y, x)=(g \cdot y, x)$.

By $h$-cobordism theorem, $D^{2} \times X$ is a differentiable disc. If we restrict the action to the boundary of $D^{2} \times X$, we get a circle group action on $S^{n}$ with its orbit space diffeomorphic to $X$ and its fixed point set, $F$, diffeomorphic to $\partial X$.

THEOREM II. For $n \geqq 5$, every differentiable circle group action on $S^{n}$ with $\operatorname{dim} F=n-2$ is differentiably equivalent to one and only one of the examples constructed above. 
Proof. We may assume that the given action is effective; if not, we may consider a quotient group which is again a circle group. Since the group $S^{1}$ is abelian, the principal isotropy subgroup is $\{e\}$.

By P. A. Smith theory and the assumption $\operatorname{dim} F=n-2$ we see that $F$ is an $(n-2)$-cohomology sphere. By Bochner's theorem, a differentiable action is always orthogonal around a fixed point, $x$. In our case, the representation is faithful and leaves an $(n-2)$-subspace fixed. It is easy to see that the representation is always the standard one, and hence there exists an invariant neighborhood $N$ of $F$ in $S^{n}$ such that $S^{1}$ acts freely on $N-F$.

We claim that $S^{1}$ acts freely on $S^{n}-F$, i.e., there are only two types of isotropy subgroups, namely $\{e\}$ and the whole group $S^{1}$. Suppose the contrary, then there exists a $Z_{p}$ subgroup in $S^{1}, p$ a suitable prime, with $F\left(Z_{p}, S^{n}\right) \supset F, F\left(Z_{p}, S^{n}\right) \neq F$. By the above fact that $S^{1}$ acts freely on $N-F$, we see that $F\left(Z_{p}, S^{n}\right)$ has at least two components, which is clearly a contradiction to $\mathrm{P}$. A. Smith theory that $F\left(Z_{p}, S^{n}\right)$ is a $Z_{p}$-homology sphere.

It follows from the fact that $S^{1}$ acts freely on $S^{n}-F$, that the associated orbit space $X$ is a manifold with boundary $\partial X=\pi(F), \pi$ the projection map. Moreover, we may blow up $S^{n}$ along $F$ to get a manifold with boundary $(M, \partial M)$ such that $S^{1}$ acts freely on $(M, \partial M)$ and the following diagram is naturally defined and commutative [4]:

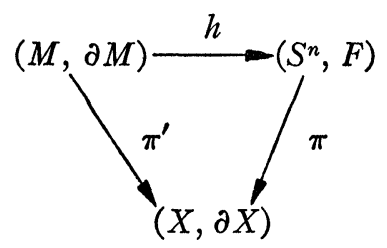

where $h$ is an equivariant relative diffeomorphism and $\pi, \pi^{\prime}$ are projections onto their common orbit spaces $(X, \partial X)$.

Since $S^{1} \rightarrow M \rightarrow X$ is a fibration, we have

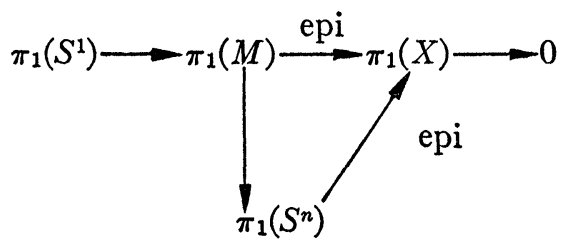

hence, $\pi_{1}(X)=0$. 
A similar argument shows that $H_{i}(X)=0$ for $i \geqq 1$ and hence $X$ is compact contractible and the fibration

$$
S^{1} \rightarrow M \rightarrow X
$$

must be trivial, i.e., $(M, \partial M)=\left(S^{1} \times X, S^{1} \times \partial X\right)$. By the construction of $(M, \partial M),\left(S^{n}, F\right)$ may be obtained from $(M, \partial M)=\left(S^{1} \times X\right.$, $\left.S^{1} \times \partial X\right)$ by identifying every circle $S^{1} \times\{x\} ; x \in \partial X$ to a point, which is equal to $\left(\partial\left(D^{2} \times X\right),\{0\} \times \partial X\right)$ up to diffeomorphism. q.e.d.

Theorem I' follows immediately from Theorem II. The unsettled cases $n=4,5$ corresponding to the unsolved Poincaré conjecture for the dimensions 3,4 .

In the cases $n=5,3$ Theorem I follows from the same argument and the fact that $S^{5}-F=M-\partial M=S^{1} \times(X-\partial X)$ is of the same homotopy type as $S^{1}$; then apply a result of J. Stallings [9].

REMARK. The case $n=4$ is the only unsettled case but it is implied by the proof that $\pi_{1}\left(S^{4}-F\right)=Z$. This shows that an example with similar properties of the example of Mazur [5] is impossible.

\section{REFERENCES}

1. A. Borel, Seminar on transformation groups, Annals of Mathematic Studies No. 46, Princeton Univ. Press, Princeton, N. J., 1960.

2. R. H. Fox, On knots whose points are fixed under a periodic transformation of the 3-sphere, Osaka Math. J. 10 (1958), 31-35.

3. C. H. Giffen, Periodic sphere transformations with knotted fixed point sets, Notices Amer. Math. Soc. 11 (1964), 341.

4. W.-Y. Hsiang, On the classification of $S O(n)$ actions on simply connected $\pi$-manifolds of dimension less than $2 n-1$ (to appear).

5. B. Mazur, Symmetric homology spheres, Illinois J. Math. 6 (1962), 245-250.

6. - Corrections to my paper, "Symmetric homology spheres," Illinois J. Math. 8 (1964), 175.

7. D. Montgomery and L. Zippin, Topological transformation groups, Interscience, New York, 1955.

8. P. A. Smith, Transformation of finite period. II, Ann. of Math. (2) 40 (1939), 690-711.

9. J. Stallings, On topologically unknotted spheres, Ann. of Math. (2) 77 (1963), 490-503.

Princeton UnIVERSITY 